\title{
METHOD ADJUSTMENT AND EQUATION SET OF MAXIMUM RAINFALL INTENSITY, DURATION AND FREQUENCY IN THE MATO GROSSO DO SUL STATE ${ }^{1}$
}

\author{
SILVIO B. PEREIRA ${ }^{1}$, CARLOS P. Z. NÓIA ${ }^{2}$, RAFAEL A. ALMEIDA ${ }^{3}$, \\ CLÍVIA D. COELHO
}

\begin{abstract}
Due to the lack of information concerning maximum rainfall equations for most locations in Mato Grosso do Sul State, the alternative for carrying out hydraulic work projects has been information from meteorological stations closest to the location in which the project is carried out. Alternative methods, such as 24 hours rain disaggregation method from rainfall data due to greater availability of stations and longer observations can work. Based on this approach, the objective of this study was to estimate maximum rainfall equations for Mato Grosso do Sul State by adjusting the 24 hours rain disaggregation method, depending on data obtained from rain gauge stations from Dourado and Campo Grande. For this purpose, data consisting of 105 rainfall stations were used, which are available in the ANA (Water Resources Management National Agency) database. Based on the results we concluded: the intense rainfall equations obtained by pluviogram analysis showed determination coefficient above 99\%; and the performance of 24 hours rain disaggregation method was classified as excellent, based on relative average error WILMOTT concordance index (1982).
\end{abstract}

KEYWORDS: intense rainfall, rainfall disaggregation, weather stations.

\section{AJUSTE DE METODOLOGIA E DETERMINAÇÃO DAS EQUAÇÕES DE INTENSIDADE, DURAÇÃO E FREQUÊNCIA DA PRECIPITAÇÃO PLUVIAL MÁXIMA PARA O ESTADO DE MATO GROSSO DO SUL}

RESUMO: Devido à carência de informações relativas às equações de precipitação máxima para a maioria das localidades do Estado de Mato Grosso do Sul, a alternativa para a realização de projetos de obras hidráulicas tem sido utilizar informações de postos meteorológicos mais próximos da localidade na qual o projeto é realizado ou fazer uso de metodologias alternativas, tal como o método de desagregação de chuvas de 24 horas, a partir de dados pluviométricos em função da maior disponibilidade de estações e maior tempo de observações. Com base nesse enfoque, o objetivo do trabalho foi estimar as equações de precipitação máxima para o Estado de Mato Grosso do Sul por meio do ajuste da metodologia de desagregação de chuvas de 24 horas, em função dos dados obtidos das estações pluviográficas de Dourados e de Campo Grande. Para tanto, foram utilizados dados consistidos em 105 estações pluviométricos, disponíveis no banco de dados da ANA. Com base nos resultados, foi possível concluir: as equações de chuvas intensas obtidas pela análise de pluviogramas apresentaram coeficiente de determinação acima de 99\%; e o desempenho do ajuste da metodologia de desagregação de chuvas de 24 horas foi classificado como ótimo, com base no erro médio relativo ao índice de concordância de WILMOTT (1982).

PALAVRAS-CHAVE: chuvas intensas, desagregação de chuvas, estações meteorológicas.

\footnotetext{
${ }^{1}$ This paper is part of the second author's Masters Thesis financed by CNPq.

${ }^{2}$ Universidade Federal de Viçosa - UFV

${ }^{3}$ Universidade Federal da Grande Dourados - UFGD

${ }^{4}$ Universidade Federal de Viçosa - UFV

${ }^{5}$ Universidade Federal de Viçosa - UFV

Recebido pelo Conselho Editorial em: 9-9-2013

Aprovado pelo Conselho Editorial em: 3-3-2014
} 


\section{INTRODUCTION}

Knowledge of the quantities characterizing a rainfall that relates the intensity, duration and frequency (IDF), also known as intense rainfalls equation, shows great technical interest in hydraulic structures projects, such as weir dimensioning, watercourse rectification, sewers, drainage systems, among others (PRUSKI et al., 2006; SANTOS et al., 2009).

A research, performed under an agreement between the Sanitation Company of Minas Gerais (COPASA) and the Federal University of Viçosa (UFV), under the Support Foundation of UFV (FUNARBE), set theoretical models of probability distribution to the intense rainfall data for 193 rain gauge stations in Minas Gerais State and at boundaries between Bahia and Espírito Santo States. The adjusted parameters of intensity, duration and frequency equations showed high variability; thus, demonstrating the importance of obtaining intense rain equation for each location (FREITAS et al., 2001).

Due to the lack of information concerning intense rain equations for most Brazilian locations, an alternative to perform hydraulic structure projects has been using information from weather stations nearest the location in which the project is carried out. This procedure, however, can lead to unreliable estimates (PEREIRA et al., 2007; SILVA et al., 2003; SILVA et al., 2002).

Some methods have been developed in Brazil to obtain shorter rains from daily rainfall records, due to existence of a vast rainfall network installed, mainly to serve power generation sector. The methods use multiplicative coefficients, among which the 24 hours rain disaggregation method stands out (CETESB, 1986; OLIVEIRA et al., 2008).

Studies conducted based on annual pluviometric and rain gauge data series, observed simultaneously in São Paulo, showed that average rain heights, of one or two days, can be considered a fairly representative estimate of 24-hour-rainfall of same frequency. They also observed that regardless of return period, there is a constant relationship between 24-hour-rainfalls and one-day-rainfalls, whose value was 1.14 (PINTO et al., 1996).

GENOVEZ \& ZUFFO (2000), cited by SANTOS et al. (2009), however, concluded that the methods based on the relationship among intense rainfall of different durations have regional validity. According to the authors, it is convenient to establish new coefficients that are related to local microclimates, for local estimates.

Besides the intense rainfall equation method, it is of fundamental importance to adjust methods that enable equation estimations from daily rainfall data due to greater availability of pluviometric stations and longer observations, so that maximum average rainfall intensity for locations out of rain gauge stations can estimated accurately. However, there are several works with direct application of alternative methods, such as FIETZ et al. (2010); FIETZ \& COMUNELLO (2007); GARCIA (2010); OLIVEIRA et al. (2008); and SANTOS et al. (2009).

Based on this approach, the objectives of this work were to obtain the maximum rainfall equations for the cities of Dourado and Campo Grande, located in Mato Grosso do Sul State. In addition, determine intensity, duration and frequency equations by fitting 24-hour rain disaggregation method into data obtained by Dourados and Campo Grande.

\section{MATERIAL AND METHODS}

We used the rainfall data from two weather stations available for Dourados-MS, one operated by Embrapa Western Agriculture and another by the Federal University of Grande Dourados (UFGD) in this work. For the region of Campo Grande a weather station belonging to the National Institute of Meteorology (INMET) was used. Table 1 shows the geographical coordinates of the stations and data collection periods. 
TABLE 1. Geographical coordinates of the weather stations and collection period of rainfall data used in the estimation of maximum average rainfall for Dourados-MS and Campo Grande-MS, Brazil.

\begin{tabular}{|c|c|c|c|c|}
\hline City & Weather station & Latitude & Longitude & Period \\
\hline \multirow{2}{*}{ Dourados: } & Embrapa (CPAO) & $22^{\circ} 16^{\prime} S$ & $54^{\circ} 49^{\prime} \mathrm{W}$ & $\begin{array}{l}1979-1990 \\
1991-2004\end{array}$ \\
\hline & Universidade Federal da Grande Dourados (UFGD) & $22^{\circ} 11^{\prime} S$ & $54^{\circ} 55^{\prime} \mathrm{W}$ & $2004-2010$ \\
\hline Campo Grande: & Instituto Nacional de Meteorologia (INMET) & $20^{\circ} 27^{\prime} \mathrm{S}$ & $54^{\circ} 38^{\prime} \mathrm{W}$ & $\begin{array}{l}1973-1985 \\
1993-1998 \\
2000-2009\end{array}$ \\
\hline
\end{tabular}

The equation used to relate rainfall intensity, duration and frequency showed the form described by VILLELA \& MATTOS (1975).

The phase of the rainfall data acquisition started with the selection of pluviograms containing the most torrential rains by each year. Maximum annual rainfalls were determined for durations of 10, 30, 60, 120, 360 and 1440 min. By dividing the maximum rainfall heights with the corresponding duration, we obtained maximum annual rainfall intensity for each duration.

Annual maximum intensity series for each duration were adjusted to the Gumbel extreme value distribution. The distribution parameters were estimated by maximum likelihood method and data adherence to the theoretical distribution assessed by Kolmogorov-Smirnov test at 20\% probability.

Using non-linear adjustment technique based on maximum annual rainfall intensity values, in return periods 2, 5, 10, 20, 50 and 100 years and the different studied durations, we obtained IDF equation parameters.

To compare the 24-hour rainfall disaggregation method with the pluviogram analysis method (standard method); primary the first method was used without changing multiplicative coefficients, as proposed by the Department of Electricity and Water / Basic Sanitation and Technology Center (DAEE / CETESB 1980), in the cities of Dourados and Campo Grande. This method adopts an average factor of 1.14 to convert maximum rainfall into 1 day, that is, from Gumbel distribution method into 24 hour-rainfall; for the remaining rainfall duration times, coefficients are employed according to the aforementioned method, allowing to generate enough points to define IDF equation related to different return periods.

Settings of probability distributions, such as completion of adhesion test, were made similarly to data obtained by pluviogram analysis method, as described by PEREIRA et al. (2007).

Results obtained by intense rainfall equations adjusted by 24-hour rainfall disaggregation method were compared with those obtained by the pluviogram analysis. Method performance was evaluated by relative error and concordance index proposed by WILLMOTT (1981). We also used performance analysis criterion proposed by CAMARGO \& SENTELHAS (1997).

For adjusting the 24-hour rainfall disaggregation, and therefore obtaining the relationship between rainfall heights by each duration, rainfall height values in different durations were divided by maximum 24-hour rainfall height. Therefore, coefficients, which allowed conversion of the local 24-hour rainfall height with durations of 10,30,60,120, 360 and $1440 \mathrm{~min}$, were obtained. Multiplicative coefficients representing each duration were obtained by geometric mean of the analyzed period.

Performance of alternative method adjustment was also assessed using relative mean error, concordance index (WILMOTT, 1982) and performance analysis model (CAMARGO e SENTELHAS, 1997). 
After adjusting alternative method, we proceeded to implementation. Then, we selected historical data series of daily rainfall from pluviometric stations located in the State, which were obtained from Hydrological Information System of the National Water Agency (Hidroweb) (ANA, 2013). Subsequently, data were subjected to a consistency analysis and locations whose series had less than 12 years of complete observations were eliminated, resulting in 105 pluviometric stations (Figure 1).

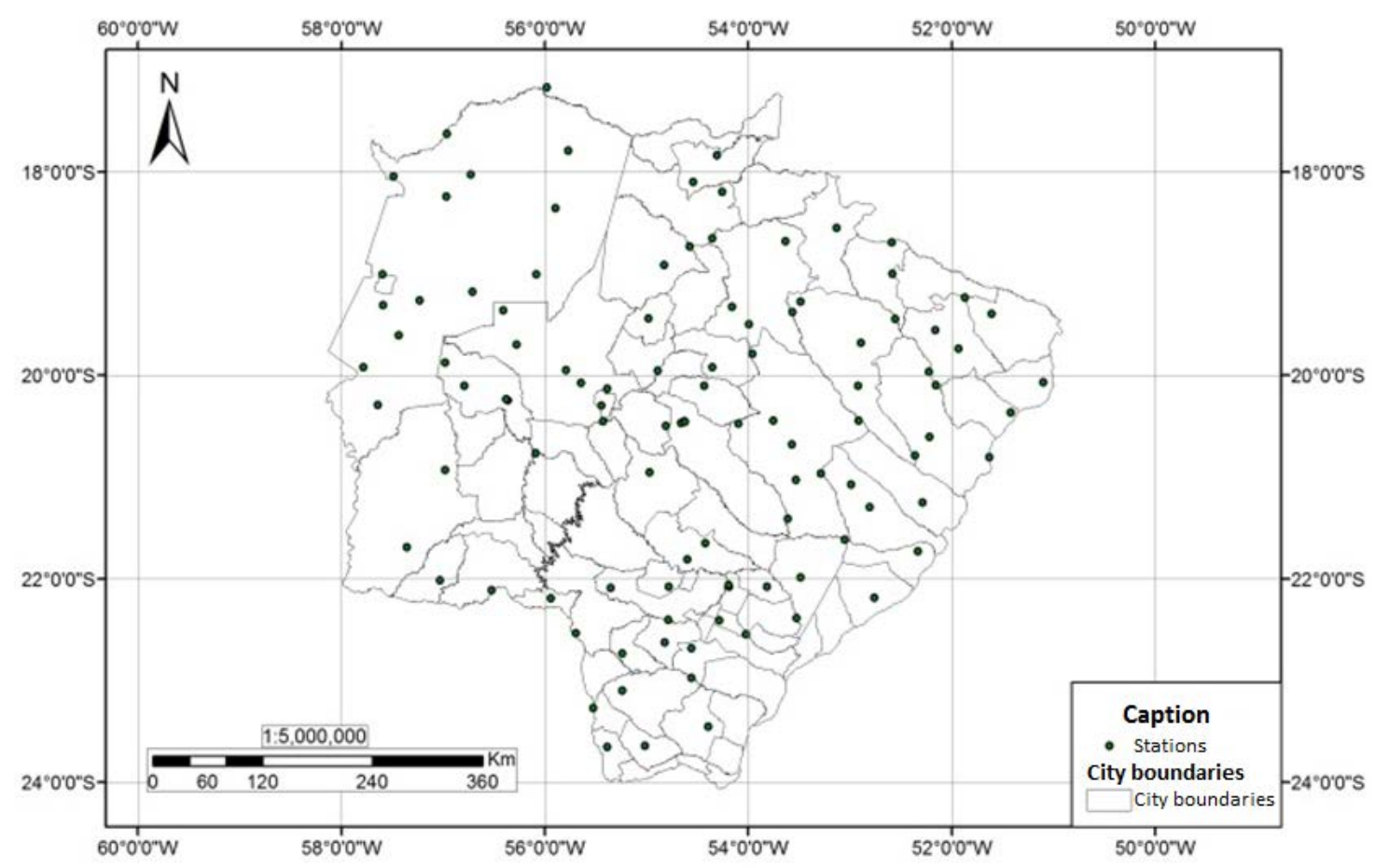

FIGURE 1. Spatial distribution of rainfall stations used in the study, in Mato Grosso do Sul State, Brazil.

For each pluviometric station the historical series of maximum annual values were set by applying multiplicative coefficients adjusted for the 24-hour rainfall disaggregation method and, after the procedure described above, obtaining the IDF equations of rainfall for each pluviometric station.

For spatial distribution of rainfall intensity data expected in Mato Grosso do Sul, we used ArcGIS software with the inverse interpolation of power distance (IPD), considering a 20-year return period, with 30 and 1440 min durations. Although agricultural projects often make hydrological predictions with a 10-year return period (SCHWAB et al., 1966, citado por PRUSKI, 2009; CRUCIANI, 1980), in studies in Southern Minas Gerais, the most advisable return period to implement hydroagricultural projects was 20 years (EUCLYDES, 1987).

\section{RESULTS AND DISCUSSION}

Figure 2 shows the frequency distribution of maximum annual rainfall observed and estimated using Gumbel model for Dourados-MS and Campo Grande, respectively. We might see that Gumbel distribution in the studied areas was adequate to estimate maximum rainfall, where there is good adhesion between observed and theoretical distribution.

Once Gumbel model adherence was verified, maximum annual intensities for 10, 30, 60, 120, 360 and 1440 minutes were estimated, considering 2, 5, 10, 20, 50 and 100 year return period. Table 2 presents the IDF equations for Dourados-MS and Campo Grande-MS, which relates duration and return period with maximum rainfall intensity expected, their respective determination coefficients and period analyzed. 
A.

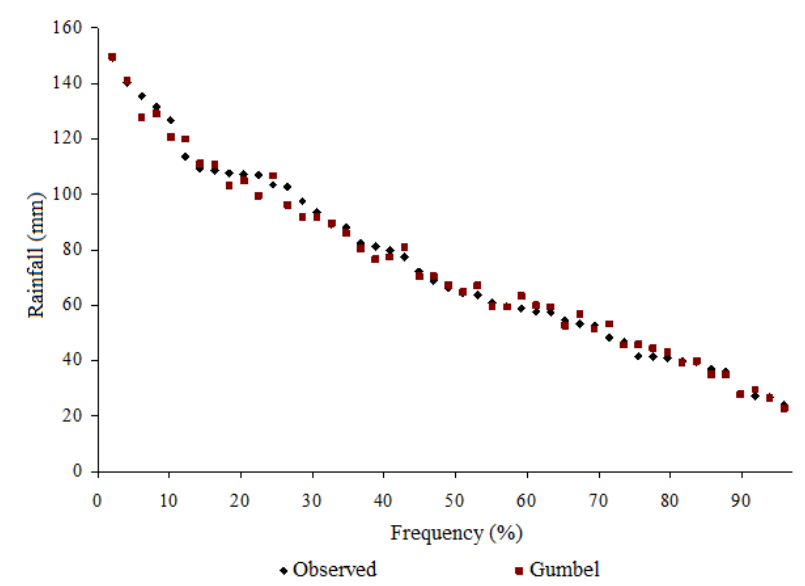

B.

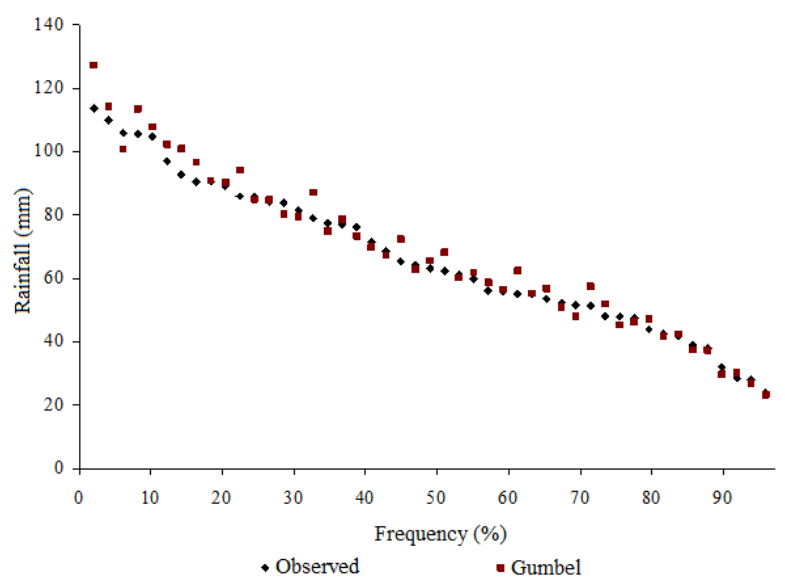

FIGURE 2. Frequency distribution of observed and estimated maximum rainfall by Gumbel model, wherein: a) Dourados-MS, Brazil; and b) Campo Grande-MS, Brazil.

Analyzing determination coefficient $\left(r^{2}\right)$ in Table 2, we observed that generated equations allow calculating maximum rainfall intensity at different durations and return periods, with great accuracy.

TABLE 2. Intensity maximum average rainfall equations $\left(\mathrm{i}_{\mathrm{m}}\right)$, in $\mathrm{mm} \mathrm{h}^{-1}$; depending on the return period $(\mathrm{T})$, in years; and rainfall duration $(\mathrm{t})$, in minutes; for Dourados-MS and Campo Grande-MS, in Brazil.

\begin{tabular}{lccc}
\hline Station & Equation & $\mathrm{r}^{2}$ & Analyzed period \\
\hline Dourados & $i_{m}=\frac{2455.13 T^{0.18}}{(t+28.14)^{0.88}}$ & 0.992 & $1979-1990$ \\
& & & $1992-2010$ \\
Campo Grande & $i_{m}=\frac{2575.12 T^{0.16}}{(t+19.87)^{0.93}}$ & 0.997 & $1973-1985$ \\
& & & $1993-1998$ \\
\hline
\end{tabular}

Table 3 presents maximum rainfall intensities for 10, 30, 60, 120, 360 and 1440 minute durations within return periods of 2, 5, 10, 20, 50 and 100 years; which were obtained through pluviogram analysis (standard method), 24-hour rainfall disaggregation as proposed by DAEE/CETESB (1980), and multiplicative coefficients of the 24-hour method adjustment. They were applied to evaluate rainfall disaggregation method performance compared with standard method by means of relative mean error (EMR) and concordance index proposed by WILMOTT (1982).

The standard method achieved excellent performance with respect to Gumbel estimation, with average concordance index of 0.97 for Dourados, and amplitude from 0.94 (duration $1440 \mathrm{~min}$ ) to 0.99 (durations of 10 and $30 \mathrm{~min}$ ); and for Campo Grande, average of 0.99, and amplitude from 0.97 (duration $1440 \mathrm{~min}$ ) to 0.99 (other durations).

The alternative method (proposed by DAEE/CETESB, 1980) compared with multiplicative coefficients adjusted according to standard method; we found better performance for adjusted coefficients in relation to alternative one. They reached an average concordance index of 0.93 for Dourados (amplitudes from 0.80 to 0.99, observed in 1440 and 120 min durations, respectively) and 0.96 for Campo Grande (amplitudes from 0.93 to 0.99, in 60 and 10 min durations, respectively). 
TABLE 3. Analysis of rainfall intensities calculated by the ratio between intensity, duration and frequency, obtained by standard method (pluviograms analysis)-(IDF1); 24-hour rainfall disaggregation proposed by DAEE/CETESB (1980)-(IDF2); 24-hour rainfall disaggregation with coefficients (IDF3); Relative Mean Error (RME) compared with Gumbel method; and concordance index (d), for stations in Dourados-MS and Campo Grande-MS, Brazil.

\begin{tabular}{|c|c|c|c|c|c|c|c|c|c|c|c|}
\hline \multicolumn{12}{|c|}{ DOURADOS } \\
\hline $\begin{array}{c}\text { Duration } \\
\text { (min) }\end{array}$ & $\begin{array}{c}\mathrm{RP} \\
\text { (years) }\end{array}$ & $\begin{array}{c}\text { GUMBEL } \\
(\mathrm{mm} / \mathrm{h})\end{array}$ & $\begin{array}{c}\text { IDF1 } \\
(\mathrm{mm} / \mathrm{h})\end{array}$ & $\begin{array}{c}\text { RME1 } \\
(\%)\end{array}$ & $\mathrm{d}$ & $\begin{array}{c}\text { IDF2 } \\
(\mathrm{mm} / \mathrm{h})\end{array}$ & $\begin{array}{c}\text { RME2 } \\
(\%)\end{array}$ & $\bar{d}$ & $\begin{array}{c}\text { IDF3 } \\
(\mathrm{mm} / \mathrm{h})\end{array}$ & $\begin{array}{c}\text { RME3 } \\
(\%)\end{array}$ & $\bar{d}$ \\
\hline 10 & 2 & 106.48 & 111.29 & 4.52 & \multirow{6}{*}{$\begin{array}{c}0.99 \\
\text { Great }\end{array}$} & 85.47 & -23.20 & \multirow{6}{*}{$\begin{array}{c}0.93 \\
\text { Great }\end{array}$} & 116.81 & 4.96 & \multirow{6}{*}{$\begin{array}{c}0.91 \\
\text { Great }\end{array}$} \\
\hline 10 & 5 & 137.58 & 131.22 & -4.63 & & 105.53 & -19.57 & & 142.50 & 8.60 & \\
\hline 10 & 10 & 158.18 & 148.63 & -6.04 & & 123.78 & -16.72 & & 165.62 & 11.43 & \\
\hline 10 & 20 & 177.93 & 168.35 & -5.39 & & 145.18 & -13.76 & & 192.50 & 14.34 & \\
\hline 10 & 50 & 203.50 & 198.49 & -2.46 & & 179.26 & -9.69 & & 234.83 & 18.31 & \\
\hline 10 & 100 & 222.67 & 224.83 & 0.97 & & 210.25 & -6.48 & & 272.94 & 21.40 & \\
\hline 30 & 2 & 70.08 & 76.67 & 9.40 & \multirow{6}{*}{$\begin{array}{c}0.99 \\
\text { Great }\end{array}$} & 51.52 & -32.80 & & 77.78 & 1.45 & \\
\hline 30 & 5 & 91.37 & 90.40 & -1.06 & & 63.61 & -29.63 & & 94.89 & 4.96 & \\
\hline 30 & 10 & 105.47 & 102.39 & -2.91 & & 74.61 & -27.13 & 0.79 & 110.28 & 7.71 & 0.94 \\
\hline 30 & 20 & 118.99 & 115.98 & -2.53 & & 87.51 & -24.54 & Very & 128.18 & 10.52 & Great \\
\hline 30 & 50 & 136.49 & 136.74 & 0.19 & & 108.05 & -20.98 & good & 156.37 & 14.35 & \\
\hline 30 & 100 & 149.60 & 154.89 & 3.54 & & 126.74 & -18.18 & & 181.74 & 17.34 & \\
\hline 60 & 2 & 45.77 & 53.08 & 15.97 & & 34.32 & -35.34 & & 51.62 & -2.75 & \\
\hline 60 & 5 & 64.84 & 62.58 & -3.49 & & 42.37 & -32.29 & & 62.97 & 0.63 & \\
\hline 60 & 10 & 77.47 & 70.89 & -8.50 & 0.96 & 49.70 & -29.89 & 0.75 & 73.19 & 3.26 & 0.97 \\
\hline 60 & 20 & 89.58 & 80.29 & -10.37 & Great & 58.29 & -27.40 & Good & 85.07 & 5.95 & Great \\
\hline 60 & 50 & 105.26 & 94.67 & -10.07 & & 71.98 & -23.97 & & 103.78 & 9.63 & \\
\hline 60 & 100 & 117.01 & 107.23 & -8.36 & & 84.42 & -21.27 & & 120.62 & 12.49 & \\
\hline 120 & 2 & 26.65 & 33.54 & 25.88 & & 21.92 & -34.64 & & 30.70 & -8.47 & \\
\hline 120 & 5 & 38.29 & 39.55 & 3.30 & & 27.07 & -31.56 & & 37.46 & -5.29 & \\
\hline 120 & 10 & 45.99 & 44.80 & -2.60 & 0.98 & 31.75 & -29.13 & 0.76 & 43.54 & -2.82 & 0.99 \\
\hline 120 & 20 & 53.39 & 50.74 & -4.95 & Great & 37.24 & -26.61 & Very & 50.60 & -0.28 & Great \\
\hline 120 & 50 & 62.95 & 59.83 & -4.97 & & 45.98 & -23.15 & Good & 61.73 & 3.18 & \\
\hline 120 & 100 & 70.12 & 67.76 & -3.36 & & 53.93 & -20.42 & & 71.74 & 5.87 & \\
\hline 360 & 2 & 10.56 & 14.32 & 35.58 & & 10.30 & -28.08 & & 11.56 & -19.27 & \\
\hline 360 & 5 & 15.33 & 16.88 & 10.11 & & 12.71 & -24.69 & & 14.10 & -16.47 & \\
\hline 360 & 10 & 18.49 & 19.12 & 3.42 & 0.98 & 14.91 & -22.01 & 0,87 & 16.39 & -14.29 & 0.94 \\
\hline 360 & 20 & 21.52 & 21.66 & 0.65 & Great & 17.49 & -19.24 & Ótimo & 19.05 & -12.05 & Great \\
\hline 360 & 50 & 25.44 & 25.54 & 0.37 & & 21.60 & -15.43 & & 23.24 & -9.00 & \\
\hline 360 & 100 & 28.38 & 28.92 & 1.92 & & 25.33 & -12.43 & & 27.01 & -6.62 & \\
\hline 1440 & 2 & 2.94 & 4.42 & 50.44 & & 3.84 & -13.01 & & 3.12 & -29.42 & \\
\hline 1440 & 5 & 4.37 & 5.21 & 19.11 & & 4.74 & -8.91 & & 3.80 & -26.98 & \\
\hline 1440 & 10 & 5.32 & 5.90 & 10.81 & 0.94 & 5.57 & -5.67 & 0,99 & 4.42 & -25.07 & 0.80 \\
\hline 1440 & 20 & 6.24 & 6.68 & 7.15 & Great & 6.53 & -2.32 & Ótimo & 5.14 & -23.11 & Very \\
\hline 1440 & 50 & 7.42 & 7.88 & 6.22 & & 8.06 & 2.29 & & 6.27 & -20.44 & good \\
\hline 1440 & 100 & 8.30 & 8.92 & 7.49 & & 9.45 & 5.92 & & 7.29 & -18.37 & \\
\hline & & & & & MPO GR & NDE & & & & & \\
\hline $\begin{array}{c}\begin{array}{c}\text { Duration } \\
\text { (min) }\end{array} \\
\end{array}$ & $\begin{array}{c}\mathrm{RP} \\
\text { (years) }\end{array}$ & $\begin{array}{c}\text { GUMBEL } \\
(\mathrm{mm} / \mathrm{h})\end{array}$ & $\begin{array}{c}\text { IDF1 } \\
(\mathrm{mm} / \mathrm{h})\end{array}$ & $\begin{array}{c}\text { RME1 } \\
(\%)\end{array}$ & $\mathrm{d}$ & $\begin{array}{c}\text { IDF2 } \\
(\mathrm{mm} / \mathrm{h})\end{array}$ & $\begin{array}{c}\text { RME2 } \\
(\%)\end{array}$ & $\mathrm{d}$ & $\begin{array}{c}\text { IDF3 } \\
(\mathrm{mm} / \mathrm{h})\end{array}$ & $\begin{array}{c}\text { RME3 } \\
(\%)\end{array}$ & $\bar{d}$ \\
\hline 10 & 2 & 108.54 & 123.25 & 13.55 & & 88.07 & -28.55 & & 130.05 & 5.52 & \\
\hline 10 & 5 & 140.34 & 142.56 & 1.58 & & 101.76 & -28.62 & & 148.39 & 4.09 & \\
\hline 10 & 10 & 161.39 & 159.15 & -1.39 & 0.99 & 113.51 & -28.68 & 0.65 & 163.97 & 3.03 & 0.99 \\
\hline 10 & 20 & 181.59 & 177.68 & -2.15 & Great & 126.62 & -28.74 & Medium & 181.18 & 1.97 & Great \\
\hline 10 & 50 & 207.73 & 205.51 & -1.06 & & 146.30 & -28.81 & & 206.73 & 0.59 & \\
\hline 10 & 100 & 227.31 & 229.43 & 0.93 & & 163.20 & -28.87 & & 228.43 & -0.44 & \\
\hline 30 & 2 & 75.20 & 76.63 & 1.91 & & 53.08 & -30.73 & & 86.31 & 12.63 & \\
\hline 30 & 5 & 92.59 & 88.64 & -4.26 & & 61.33 & -30.81 & & 98.48 & 11.11 & \\
\hline 30 & 10 & 104.10 & 98.96 & -4.94 & 0.99 & 68.42 & -30.86 & 0.63 & 108.82 & 9.97 & 0.96 \\
\hline 30 & 20 & 115.14 & 110.47 & -4.05 & Great & 76.32 & -30.92 & Medium & 120.24 & 8.84 & Great \\
\hline 30 & 50 & 129.43 & 127.78 & -1.28 & & 88.18 & -30.99 & & 137.20 & 7.37 & \\
\hline 30 & 100 & 140.15 & 142.65 & 1.79 & & 98.36 & -31.05 & & 151.60 & 6.27 & \\
\hline 60 & 2 & 48.14 & 49.52 & 2.87 & & 35.35 & -28.61 & & 57.03 & 15.16 & \\
\hline 60 & 5 & 58.69 & 57.28 & -2.40 & & 40.85 & -28.68 & & 65.07 & 13.61 & \\
\hline 60 & 10 & 65.67 & 63.95 & -2.63 & 0.99 & 45.57 & -28.74 & 0.65 & 71.90 & 12.44 & 0.93 \\
\hline 60 & 20 & 72.37 & 71.39 & -1.36 & Great & 50.83 & -28.80 & Medium & 79.45 & 11.29 & Great \\
\hline 60 & 50 & 81.05 & 82.57 & 1.88 & & 58.73 & -28.87 & & 90.66 & 9.79 & \\
\hline 60 & 100 & 87.54 & 92.18 & 5.30 & & 65.51 & -28.93 & & 100.17 & 8.67 & \\
\hline 120 & 2 & 28.30 & 29.46 & 4.07 & & 22.58 & -23.34 & & 33.70 & 14.40 & \\
\hline 120 & 5 & 34.97 & 34.07 & -2.56 & & 26.09 & -23.42 & & 38.45 & 12.85 & \\
\hline 120 & 10 & 39.38 & 38.04 & -3.41 & 0.99 & 29.10 & -23.49 & 0.72 & 42.49 & 11.70 & 0.94 \\
\hline 120 & 20 & 43.61 & 42.46 & -2.63 & Great & 32.46 & -23.55 & Good & 46.94 & 10.55 & Great \\
\hline 120 & 50 & 49.09 & 49.12 & 0.06 & & 37.51 & -23.63 & & 53.57 & 9.06 & \\
\hline 120 & 100 & 53.19 & 54.83 & 3.09 & & 41.84 & -23.69 & & 59.19 & 7.94 & \\
\hline 360 & 2 & 11.22 & 11.66 & 3.93 & & 10.60 & -9.11 & & 12.51 & 7.23 & \\
\hline 360 & 5 & 14.17 & 13.49 & -4.76 & & 12.25 & -9.21 & & 14.27 & 5.78 & \\
\hline 360 & 10 & 16.12 & 15.06 & -6.54 & 0.99 & 13.66 & -9.28 & 0.95 & 15.77 & 4.70 & 0.99 \\
\hline 360 & 20 & 17.99 & 16.82 & -6.50 & Great & 15.24 & -9.35 & Great & 17.43 & 3.62 & Great \\
\hline 360 & 50 & 20.40 & 19.45 & -4.68 & & 17.61 & -9.45 & & 19.88 & 2.22 & \\
\hline 360 & 100 & 22.22 & 21.71 & -2.27 & & 19.65 & -9.52 & & 21.97 & 1.18 & \\
\hline 1440 & 2 & 3.35 & 3.35 & -0.06 & & 3.95 & 18.12 & & 3.14 & -6.25 & \\
\hline 1440 & 5 & 4.20 & 3.87 & -7.83 & & 4.57 & 17.99 & & 3.58 & -7.52 & \\
\hline 1440 & 10 & 4.77 & 4.32 & -9.28 & 0.97 & 5.10 & 17.90 & 0.86 & 3.96 & -8.46 & 0.94 \\
\hline 1440 & 20 & 5.31 & 4.83 & -9.04 & Great & 5.69 & 17.80 & Great & 4.37 & -9.40 & Great \\
\hline 1440 & 50 & 6.01 & 5.58 & -7.05 & & 6.57 & 17.68 & & 4.99 & -10.62 & \\
\hline 1440 & 100 & 6.53 & 6.23 & -4.57 & & 7.33 & 17.58 & & 5.51 & -11.54 & \\
\hline
\end{tabular}


Table 4 lists multiplicative coefficients adjusted for application of the 24-hour rainfall disaggregation method to be practiced in Mato Grosso do Sul. The results obtained showed great performance based on relative mean error (RME), concordance index, and model performance analysis criterion (Table 3).

TABLE 4. Proposition of rainfall disaggregation coefficients of rain data for Mato Grosso do Sul State, Brazil.

\begin{tabular}{lcccccc}
\hline Duration & $1440 \mathrm{~min} / 24 \mathrm{~h}$ & $720 \mathrm{~min} / 24 \mathrm{~h}$ & $360 \mathrm{~min} / 24 \mathrm{~h}$ & $240 \mathrm{~min} / 24 \mathrm{~h}$ & $180 \mathrm{~min} / 24 \mathrm{~h}$ & $120 \mathrm{~min} / 24 \mathrm{~h}$ \\
Coefficient & 1.14 & 0.94 & 0.88 & 0.82 & 0.79 & 0.74 \\
\hline Duration & $60 \mathrm{~min} / 24 \mathrm{~h}$ & $50 \mathrm{~min} / 24 \mathrm{~h}$ & $40 \mathrm{~min} / 24 \mathrm{~h}$ & $30 \mathrm{~min} / 24 \mathrm{~h}$ & $20 \mathrm{~min} / 24 \mathrm{~h}$ & $10 \mathrm{~min} / 24 \mathrm{~h}$ \\
Coefficient & 0.63 & 0.60 & 0.55 & 0.49 & 0.39 & 0.24 \\
\hline
\end{tabular}

The 24-hour rainfall disaggregation method proposed by DAEE/CETESB (1980) resulted, in general, in an underestimation of maximum rainfall intensity for both locations, except for 1440 min duration, which showed a differentiated behavior. This fact provides less security for hydroagricultural projects but, on the other hand, it decreases cost on project implementation.

The adjusted coefficients were used to estimate equation parameters that relate intensity, duration and frequency for various locations in Mato Grosso do Sul State (Table 5). There was good adjustment of intense rainfalls equations, with $r^{2}$ values above 0.98 .

We found a significant variation within the fitted equation parameters. $\boldsymbol{K}$ parameter presented a variation of $84.5 \%$, ranging from 4905.22, in Ribas do Rio Pardo town (Code: 2153002), to 9048.45 in Cassilândia town (Code: 1951004). $\boldsymbol{A}$ values showed a 133\% variation, with values from 0.13 (Chapadão do Sul, Code: 1852002) to 0.31 (Cassilândia, Code: 1951004).

Parameters $\boldsymbol{b}$ and $\boldsymbol{c}$ of the IDF adjusted equation presented the smallest variations, which were 3.6\% and 1.7\%, with amplitudes from 32.21 (Coxim, Code: 1855000) to 33.37 (Camapuã, Code: 1954006), and from 1.05 (Ribas do Rio Pardo, Code: 2153002) to 1.07 (Camapuã, Code: 1954006), respectively.

Parameters of rainfall intensity equation had great variability what highlights the need for local information attainment to perform studies and hydraulic projects. This fact shows that neighboring station use, for places that do not have the adjusted equation, interferes with the dimensioning safety and cost of projects (PEREIRA et al., 2007; SILVA et al., 2002;).

The results obtained with the spatial distribution of intense rainfall (Figure 3) were made only for the 20-year-return period, with durations of 30 and $1440 \mathrm{~min}$, because according to EUCLYDES (1987), this period is the most recommended for hydro-agricultural project implementation. Thus, the longer the return period, the greater the values of maximum intensities found and, therefore, the greater the safety and cost of the project.

We observed lower values of maximum rainfall intensities in Eastern State analyzing Figure 3 , with increasing behavior towards Western direction reaching $290 \mathrm{~mm} \mathrm{~h}^{-1}$ (20-year-return period and 30-minute duration) and $10.6 \mathrm{~mm} \mathrm{~h}^{-1}$ (1440-minute). ARAI et al. (2012) identified this same characterization for a basin located in South-Central Mato Grosso do Sul State. 
TABLE 5. Maximum rainfall intensity equation coefficients $\mathrm{K}$, a, b and c for 105 rainfall stations located in Mato Grosso do Sul State and respective determination coefficients (r2).

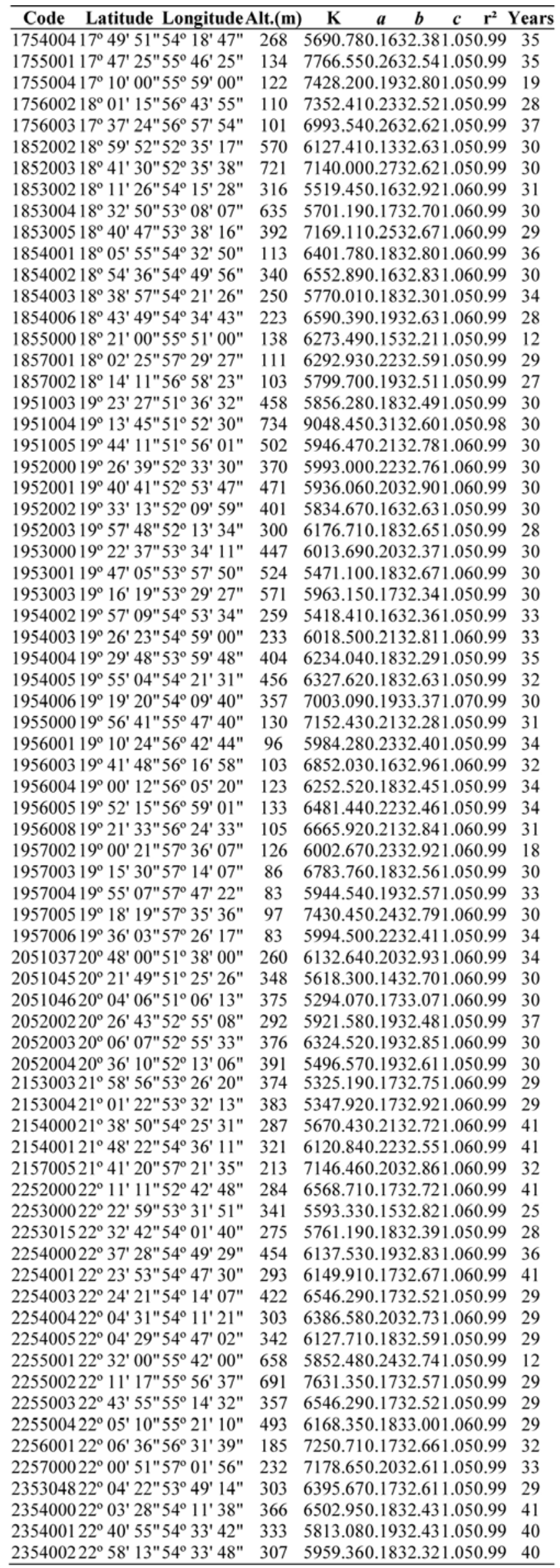

\begin{tabular}{lllllll}
\hline Code & Latitude LongitudeAlt.(m) & $\mathrm{K}$ & $\boldsymbol{a}$ & $\boldsymbol{b}$ & $\boldsymbol{c}$ & $\mathbf{r}^{2}$ Years \\
\hline
\end{tabular} $215700521^{\circ} 41^{\prime} 20^{\prime \prime} 57^{\circ} 21^{\prime} 35^{\prime \prime} 213 \quad 7146.460 .2032 .861 .060 .99 \quad 32$ $225200022^{\circ} 11^{\prime} 11^{\prime \prime} 52^{\circ} 42^{\prime} 48^{\prime \prime} \quad 284 \quad 6568.710 .1732 .721 .060 .99 \quad 41$ $225300022^{\circ} 22^{\prime} 59^{\prime \prime} 53^{\circ} 31^{\prime} 51^{\prime \prime} \quad 341 \quad 5593.330 .1532 .821 .060 .99 \quad 25$ $225301522^{\circ} 32^{\prime} 42^{\prime \prime} 54^{\circ} 01^{\prime} 40^{\prime \prime} \quad 275 \quad 5761.190 .1832 .391 .050 .99 \quad 28$ $225400022^{\circ} 37^{\prime} 28^{\prime \prime} 54^{\circ} 49^{\prime} 29^{\prime \prime} \quad 454 \quad 6137.530 .1932 .831 .060 .99 \quad 36$ $225400122^{\circ} 23^{\prime} 53^{\prime \prime} 54^{\circ} 47^{\prime} 30^{\prime \prime} \quad 293 \quad 6149.910 .1732 .671 .060 .99 \quad 41$ $225400322^{\circ} 24^{\prime} 21^{\prime \prime} 54^{\circ} 14^{\prime} 07^{\prime \prime} \quad 422 \quad 6546.290 .1732 .521 .050 .9929$ $225400422^{\circ} 04^{\prime} 31^{\prime \prime} 54^{\circ} 11^{\prime} 21^{\prime \prime} \quad 303 \quad 6386.580 .2032 .731 .060 .9929$ $225400522^{\circ} 04^{\prime} 29^{\prime \prime} 54^{\circ} 47^{\prime} 02^{\prime \prime} \quad 342 \quad 6127.710 .1832 .591 .050 .9929$ $225500122^{\circ} 32^{\prime} 00^{\prime \prime} 55^{\circ} 42^{\prime} 00^{\prime \prime} \quad 658 \quad 5852.480 .2432 .741 .050 .99 \quad 12$ $225500222^{\circ} 11^{\prime} 17^{\prime \prime} 55^{\circ} 56^{\prime} 37^{\prime \prime} 691 \quad 7631.350 .1732 .571 .050 .9929$ $225500322^{\circ} 43^{\prime} 55^{\prime \prime} 55^{\circ} 14^{\prime} 32^{\prime \prime} \quad 357 \quad 6546.290 .1732 .521 .050 .9929$ $225500422^{\circ} 05^{\prime} 10^{\prime \prime} 55^{\circ} 21^{\prime} 10^{\prime \prime} \quad 493 \quad 6168.350 .1833 .001 .060 .9929$ $225600122^{\circ} 06^{\prime} 36^{\prime \prime} 56^{\circ} 31^{\prime} 39^{\prime \prime} \quad 185 \quad 7250.710 .1732 .661 .050 .9932$ $225700022^{\circ} 00^{\prime} 51^{\prime \prime} 57^{\circ} 01^{\prime} 56^{\prime \prime} \quad 232 \quad 7178.650 .2032 .611 .050 .9933$ $235304822^{\circ} 04^{\prime} 22^{\prime \prime} 53^{\circ} 49^{\prime} 14^{\prime \prime} \quad 303 \quad 6395.670 .1732 .611 .050 .99 \quad 29$ $235400022^{\circ} 03^{\prime} 28^{\prime \prime} 54^{\circ} 11^{\prime} 38^{\prime \prime} \quad 366 \quad 6502.950 .1832 .431 .050 .99 \quad 41$ $235400122^{\circ} 40^{\prime} 55^{\prime \prime} 54^{\circ} 33^{\prime} 42^{\prime \prime} \quad 333 \quad 5813.080 .1932 .431 .050 .99 \quad 40$ $235400222^{\circ} 58^{\prime} 13^{\prime \prime} 54^{\circ} 33^{\prime} 48^{\prime \prime} \quad 307 \quad 5959.360 .1832 .321 .050 .99 \quad 40$ $235400423^{\circ} 26^{\prime} 59^{\prime \prime} 54^{\circ} 23^{\prime} 37^{\prime \prime} \quad 343 \quad 6350.210 .1732 .551 .050 .9928$ $235500023^{\circ} 05^{\prime} 52^{\prime \prime} 55^{\circ} 14^{\prime} 37^{\prime \prime} \quad 460 \quad 7848.900 .1733 .171 .060 .99 \quad 41$ $235500123^{\circ} 15^{\prime} 58^{\prime \prime} 55^{\circ} 31^{\prime} 28^{\prime \prime} \quad 510 \quad 6224.070 .1632 .551 .050 .9929$ $235500223^{\circ} 39^{\prime} 01^{\prime \prime} 55^{\circ} 23^{\prime} 31 " 319 \quad 6759.610 .1632 .781 .060 .9929$ $235500323^{\circ} 38^{\prime} 25^{\prime \prime} 55^{\circ} 01^{\prime} 09^{\prime \prime} \quad 371 \quad 6464.410 .1732 .441 .050 .9929$ $205200620^{\circ} 05^{\prime} 38^{\prime \prime} 52^{\circ} 09^{\prime} 37^{\prime \prime} 317 \quad 5322.640 .1832 .661,050.9929$ $205300020^{\circ} 26^{\prime} 41^{\prime \prime} 53^{\circ} 45^{\prime} 29^{\prime \prime} \quad 373 \quad 5962.360 .2332 .591,050.99 \quad 40$ $205300120^{\circ} 40^{\prime} 37^{\prime \prime} 53^{\circ} 34^{\prime} 17^{\prime \prime} \quad 345 \quad 5475.240 .1732 .571,050.9930$ $205300420^{\circ} 57^{\prime} 40^{\prime \prime} 53^{\circ} 17^{\prime} 17^{\prime \prime} \quad 298 \quad 5663.390 .1932 .811,060.9926$ $205400120^{\circ} 28^{\prime} 00^{\prime \prime} 54^{\circ} 40^{\prime} 00^{\prime \prime} \quad 559 \quad 6057.600 .1632 .451,050.99 \quad 12$ $205400520^{\circ} 29^{\prime} 35^{\prime \prime} 54^{\circ} 48^{\prime} 41^{\prime \prime} \quad 518 \quad 6581.910 .1732 .661,060.9934$ $205401020^{\circ} 27^{\prime} 00^{\prime \prime} 54^{\circ} 38^{\prime} 00^{\prime \prime} \quad 576 \quad 5775.200 .2032 .281,050.99 \quad 19$ $205401420^{\circ} 27^{\prime} 06^{\prime \prime} 54^{\circ} 37^{\prime} 39^{\prime \prime} \quad 562 \quad 5719.030 .1832 .621,050.9927$ $205401920^{\circ} 06^{\prime} 08^{\prime \prime} 54^{\circ} 26^{\prime} 05^{\prime \prime} \quad 577 \quad 5249.120 .2132 .511,050.9930$ $205402020^{\circ} 28^{\prime} 15^{\prime \prime} 54^{\circ} 05^{\prime} 48^{\prime \prime} 515 \quad 6017.020 .1932 .711,060.9929$ $205402120^{\circ} 57^{\prime} 05^{\prime \prime} 54^{\circ} 58^{\prime} 18^{\prime \prime} \quad 469 \quad 6817.430 .2032 .941,060.9929$ $205500120^{\circ} 07^{\prime} 40^{\prime \prime} 55^{\circ} 23^{\prime} 36^{\prime \prime} \quad 171 \quad 6686.770 .2132 .631,050.99 \quad 35$ $205500220^{\circ} 26^{\prime} 56^{\prime \prime} 55^{\circ} 25^{\prime} 51 " 175 \quad 6515.630 .2232 .481,050.9933$ $205500320^{\circ} 17^{\prime} 32^{\prime \prime} 55^{\circ} 26^{\prime} 43^{\prime \prime} \quad 204 \quad 6138.530 .2032 .611,050.9928$ $205500420^{\circ} 04^{\prime} 13^{\prime \prime} 55^{\circ} 38^{\prime} 43^{\prime \prime} \quad 146 \quad 7107.710 .2132 .371,050.9934$ $205600120^{\circ} 14^{\prime} 29^{\prime \prime} 56^{\circ} 22^{\prime} 06^{\prime \prime} \quad 141 \quad 6408.840 .2232 .631,060.9930$ $205600320^{\circ} 45^{\prime} 52^{\prime \prime} 56^{\circ} 05^{\prime} 35^{\prime \prime} \quad 139 \quad 6249.010 .1832 .481,050.9933$ $205600520^{\circ} 06^{\prime} 07^{\prime \prime} 56^{\circ} 47^{\prime} 43^{\prime \prime} 140 \quad 7143.010 .1933 .111,060.9932$ $205600620^{\circ} 14^{\prime} 00^{\prime \prime} 56^{\circ} 23^{\prime} 00^{\prime \prime} \quad 114 \quad 7568.590 .2332 .731,060.99 \quad 12$ $205600720^{\circ} 55^{\prime} 36^{\prime \prime} 56^{\circ} 59^{\prime} 02^{\prime \prime} \quad 192 \quad 7067.330 .1532 .481,050.9922$ $\begin{array}{lllll}205700020^{\circ} 17^{\prime} 27^{\prime \prime} 57^{\circ} 38^{\prime} 52^{\prime \prime} & 81 & 6415.420 .2232 .431,050.99 & 29\end{array}$ $215200020^{\circ} 47^{\prime} 00^{\prime \prime} 52^{\circ} 21^{\prime} 54^{\prime \prime} \quad 316 \quad 5518.750 .1632 .761,060.9941$ $215200121^{\circ} 43^{\prime} 33^{\prime \prime} 52^{\circ} 20^{\prime} 03^{\prime \prime} 293 \quad 6326.170 .1832 .661,050.9941$ $215200521^{\circ} 17^{\prime} 43^{\prime \prime} 52^{\circ} 48^{\prime} 38^{\prime \prime} 393 \quad 5786.050 .1532 .771,060.9937$ $215201421^{\circ} 14^{\prime} 58^{\prime \prime} 52^{\circ} 17^{\prime} 17^{\prime \prime} 336 \quad 5926.390 .1932 .381,050.9929$ $215201621^{\circ} 04^{\prime} 11^{\prime \prime} 52^{\circ} 59^{\prime} 37^{\prime \prime} 352 \quad 6027.250 .1932 .601,050.9926$ $215300021^{\circ} 36^{\prime} 55^{\prime \prime} 53^{\circ} 03^{\prime} 08^{\prime \prime} \quad 271 \quad 6424.370 .2232 .911,060.99 \quad 41$ $215300221^{\circ} 24^{\prime} 34^{\prime \prime} 53^{\circ} 36^{\prime} 48^{\prime \prime} \quad 335 \quad 4905.220 .2232 .221,050.99 \quad 29$ 
The decrease in the values of annual maximum intensities with increase in rainfall duration is also evident in Figure 3, which is typical for rains, since rainfall intensity tends to decrease with increased durations.

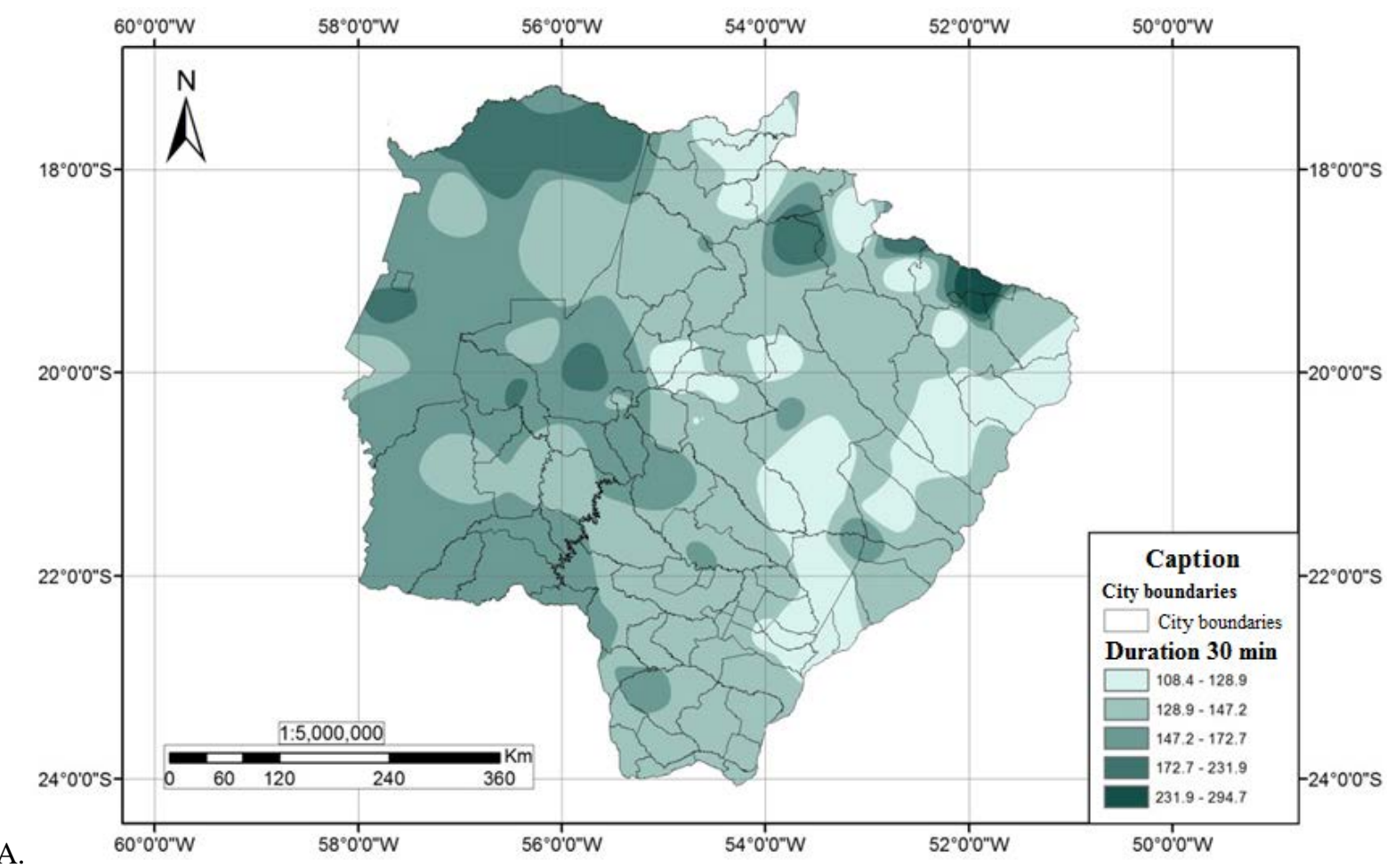

A.

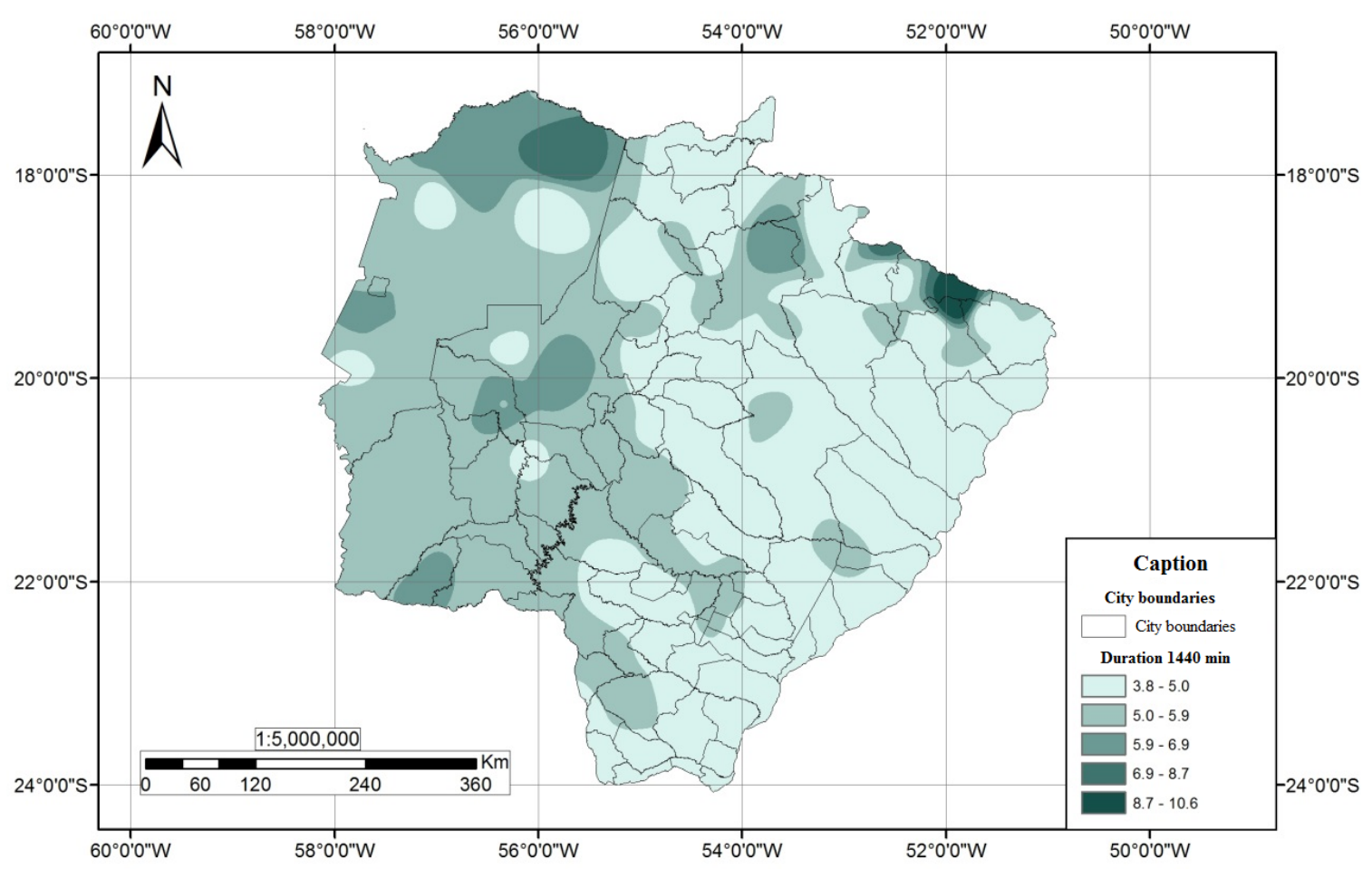

B.

FIGURE 3. Spatial distribution of maximum rainfall $\left(\mathrm{mm} \mathrm{h}^{-1}\right)$ for Mato Grosso do Sul State, with a 20-year return period, wherein: a) 30-min duration; and b) 1440-min duration. 


\section{CONCLUSIONS}

Intense rainfall equations obtained through pluviogram analysis present great adjustments with determination coefficients above 99\%;

The 24-hour rainfall disaggregation method with adjusted coefficients presented excellent performance when compared to pluviogram analysis;

The 24-hour rainfall disaggregation method with original coefficients tends to underestimate maximum rainfall intensities in Mato Grosso do Sul State, Brazil; and

The highest rainfall intensities foreseen for Mato Grosso do Sul occur in Western State and the lowest ones in Eastern area.

\section{ACKNOWLEDGEMENTS}

We thank CNPq for the financial support, as well as INMET, by disposing of the data series.

\section{REFERENCES}

ANA - Agência Nacional de Águas. HidroWeb: sistemas de Informações Hidrológicas. Disponível em: <http://hidroweb.ana.gov.br/HidroWeb>. Acesso em 15 mar. 2013.

ARAI, F.K.; PEREIRA, S.B.; GONÇALVES, G.G.G. Characterization of water availability in a hydrographic basin. Engenharia Agrícola, Jaboticabal, v.32, n.3, p.591-601, 2012.

ASSIS, F.N.; ARRUDA, H.V.; PEREIRA, A.R. Aplicações de estatística à climatologia. Pelotas: Editora Universitária, Universidade Federal de Pelotas, 1996. 161p.

CAMARGO, A.P.; SENTELHAS, P.C. Avaliação do desempenho de diferentes métodos de estimativa da evapotranspiração potencial no Estado de São Paulo. Revista Brasileira de Agrometeorologia, Santa Maria, v. 5, n. 1, p. 89-97, 1997.

CETESB - Companhia de Tecnologia de Saneamento Ambiental. Drenagem urbana: manual de projeto. São Paulo, 1986. 494p.

CRUCIANI, D.E. A drenagem na agricultura. São Paulo: Nobel, 1980. 333p.

DAEE; CETESB - Departamento de Água e Energia Elétrica; Companhia de Tecnologia de Saneamento Ambiental. Drenagem urbana: manual de projeto. São Paulo, SP. 1980. 466p.

EUCLYDES, H.P. Saneamento agrícola: atenuação das cheias; metodologia e projeto. 2.ed. Belo Horizonte: Ruralminas, 1987. 320p.

FIETZ, C.R.; COMUNELLO, E. Chuvas intensas em Mato Grosso do Sul. Dourados: Embrapa Agropecuária Oeste, 2007. 86p. (Documentos, 90).

FIETZ, C.R.; COMUNELLO, E; CREMON, C.; DALLACORT, R. PEREIRA, S.B. Chuvas intensas no Estado de Mato Grosso. Dourados, MS: Embrapa Agropecuária Oeste, 2010. 117p. (Documentos, 104).

FREITAS, A.J., SILVA, D.D., PRUSKI, F.F., PINTO, F.A., PEREIRA, S.B., GOMES FILHO, R.R., TEIXEIRA, A.F., BAENA, L.G.N., MELLO, L.T.A., NOVAES, L.F. Equações de chuvas intensas no Estado de Minas Gerais. Belo Horizonte: Companhia de Saneamento de Minas Gerais; Viçosa: Universidade Federal de Viçosa, 2001. 65p.

GARCIA, S.S. Determinação da equação de intensidade-duração-frequência da precipitação pluvial para sete estações meteorológicas da região Centro Sul do Estado de Mato Grosso. 2010. 90f. Tese (Mestrado em Engenharia Agrícola) - Universidade Federal de Mato Grosso, Cuiabá, 2010. 
OLIVEIRA, L.F.C.; ANTONINI, J.C.A.; FIOREZE, A.P.; SILVA, M.A.S. Métodos de estimativa de precipitação máxima para o Estado de Goiás. Revista Brasileira de Engenharia Agrícola e Ambiental, Campina Grande, UAEAg/UFCG, v.12, n.6, p.620-625, 2008.

PEREIRA, S.B.; FIETZ, C.R.; PEIXOTO, P.P.P.; ALVES SOBRINHO, T.; SANTOS, F.M. Equação de intensidade, duração e frequência da precipitação para a região de Dourados, MS, Dourados, MS: Embrapa Agropecuária Oeste, 2007. 18p. (Documentos, 44). Disponível em: $<$ http://www.cpao. embrapa.br/publicacoes>. Acesso em: 1 de jun. 2012.

PINTO, F.A.; FERREIRA, P.A.; PRUSKI, F.F.; ALVES, A.R.; CECON, P.R. Estimativa de chuvas intensas no Estado de Minas Gerais utilizando registros diários. Engenharia Agrícola, Jaboticabal, v.16, n.2, p.8-21, 1996.

PRUSKI, F.F. Conservação do solo e água: práticas mecânicas para o controle da erosão hídrica. 2.ed. Viçosa: Editora UFV, 2009. 279p.

PRUSKI, F.F.; SILVA, D.D.; TEIXEIRA, A.F.; CECÍLIO, R.A.; SILVA, J.M.A.; GRIEBELER, N.P. Hidros: dimensionamento de sistemas hidroagrícolas. Viçosa: Editora UFV, 2006. 259p.

SANTOS, G.G.; FIGUEIREDO, C.C.; OLIVEIRA, L.F.C.; GRIEBELER, N.P. Intensidadeduração-frequência de chuvas para o Estado de Mato Grosso do Sul. Revista Brasileira de Engenharia Agrícola e Ambiental, Campina Grande, PB, UAEA/UFCG, v.13, p.899-905, 2009. Suplemento.

SILVA, D.D.; GOMES FILHO, R.R.; PRUSKI, F.F.; PEREIRA, S.B; NOVAES, L.F. Chuvas intensas para o Estado da Bahia. Revista Brasileira de Engenharia Agrícola e Ambiental, Campina Grande, v.6, n.2, p.362-367, 2002.

SILVA, D.D.; PEREIRA, S.B; PRUSKI, F.F.; GOMES FILHO, R.R.; LANA, A.M.Q.; BAENA, L.G.N. Equações de intensidade-duração-frequência da precipitação pluvial para o Estado de Tocantins. Engenharia na Agricultura, Viçosa, MG, v.11, n.1/4, p.7-14, 2003.

VILLELA, S., MATTOS, A. Hidrologia aplicada. São Paulo: McGraw-Hill do Brasil. 1975. 245p. WILLMOTT, C.J. On the validation on models. Physical Geography,Norwich, v.2, n.2, p.184-194, 1981.

WILLMOTT, C.J. Some comments on the evaluation of model performance. Bulletin of the American Meteorological Society, Boston, v.63. n.11, p.1309-1313, 1982. 